\title{
Nuclear data activities of the EUROfusion consortium
}

Ulrich Fischer ${ }^{1, *}$, Marilena Avrigeanu ${ }^{2}$, Vlad Avrigeanu ${ }^{2}$, Alexander Konobeyev ${ }^{1}$, Ivo Kodeli ${ }^{3}$, Helmut Leeb ${ }^{4}$, Michael Fleming ${ }^{5}$, Mark Gilbert ${ }^{6}$, Dimitri Rochman ${ }^{7}$, Pavel Pereslavtsev ${ }^{1}$, Patrick Sauvan ${ }^{8}$, Georg Schnabel $^{9}$, Stanislav Simakov $^{1}$, and Henrik Sjöstrand ${ }^{9}$

${ }^{1}$ Karlsruhe Institute of Technology (KIT) Hermann-von-Helmholtz-Platz 176344 Eggenstein-Leopoldshafen Germany

${ }^{2}$ Horia Hulubei National Institute of Physics and Nuclear Engineering (IFIN-HH) RO-077125 Magurele Romania

${ }^{3}$ Jozef Stefan Institute (JSI), Jamova 39, 1000 Ljubljana Slovenia

${ }^{4}$ Technische Universität Wien, Atominstitut, Wiedner Hauptstrasse 8-10, 1040 Wien, Austria

${ }^{5}$ Nuclear Energy Agency (NEA), 46, quai Alphonse Le Gallo, 92100 Boulogne-Billancourt, France

${ }^{6}$ Culham Centre for Fusion Energy, Culham Science Centre, Abingdon, OX14 3DB United Kingdom

${ }^{7}$ Paul Scherrer Institut, 5232 Villigen PSI, Switzerland

${ }^{8}$ Universidad Nacional de Educación a Distancia, C. Juan del Rosal, 12, 28040 Madrid, Spain

${ }^{9}$ Uppsala University, Department of Physics and Astronomy, Division of Applied Nuclear Physics, 75120 Uppsala, Sweden

\begin{abstract}
The activities of the EUROfusion consortiums on the development of high quality nuclear data for fusion applications are presented. The activities, implemented in the Power Plant Physics and Technology (PPPT) programme of EUROfusion, include nuclear data evaluations for neutron and deuteron induced reactions and the production of related data libraries which satisfy the needs for nuclear analyses of the DEMO fusion power plant and the IFMIF-DONES neutron source. The activities are closely linked to the JEFF initiative of the NEA Data Bank. The evaluation work is complemented by extensive benchmark, sensitivity and uncertainty analyses to check the performance of the evaluated cross-section data and libraries against integral experiments.
\end{abstract}

\section{Introduction}

The European Fusion Programme aims at the realisation of fusion as energy source for the production of electricity, delivered to the grid, by the middle of the century [1]. The Fusion Roadmap specifies eight missions and three major facilities (ITER, DEMO and a neutron source) to achieve this goal. Related development works are conducted within the Power Plant Physics and Technology (PPPT) programme of the EUROfusion consortium. This includes, as central elements, the development of a fusion power demonstration plant (DEMO), and the high intense neutron source IFMIF-DONES (International Fusion Material Irradiation Facility- DEMO Oriented NEutron Source) for the material qualification.

Neutronics simulations play a fundamental role in the design and optimisation of these facilities, including the evaluation and verification of their nuclear performances. Accurate data are required to predict the tritium breeding capability, assess the shielding efficiency, estimate the nuclear power generated in the system, and produce activation and radiation damage data for the irradiated materials/components. Likewise this applies to the radiation dose fields to be provided after shut-down or during maintenance periods. The availability of high quality nuclear data is thus a pre-requisite for reliable design calculations affecting the nuclear design and performance of the facil-

*e-mail: ulrich.fischer@kit.edu ities, as well as safety, licensing, waste management and decommissioning issues.

The EUROfusion consortium has implemented a dedicated activity on the development of high quality nuclear data to support neutronics in the PPPT programme. This includes the evaluation of general purpose neutron cross-section data as required for design calculations using particle transport codes, the generation of new activation and displacement damage cross-section data libraries, and evaluation of deuteron cross-sections as required for the IFMIF-DONES accelerator. This work is complemented by extensive benchmark, sensitivity and uncertainty analyses to check the performance of the evaluated cross-section data and libraries against integral experiments.

This paper provides an overview of the related nuclear data activities conducted in the PPPT programme since 2017. The focus is on the achievements obtained in the area of nuclear data evaluations, benchmarking and validation, activation and radiation damage, nuclear model and method improvements, and sensitivity/uncertainty assessments.

\section{Nuclear Data in the PPPT programme}

The development of nuclear data for fusion technology in Europe has been previously organized by Fusion for Energy (F4E), Barcelona, through a framework partnership agreement with the Consortium on Nuclear Data De- 
velopment and Analysis [2]]. The related activities addressed the nuclear data needs of the key facilities ITER, IFMIF and DEMO and included the evaluation and validation of relevant nuclear cross-section data, the development/extension of codes and software tools required for nuclear model calculations and sensitivity/uncertainty assessments.

Starting in 2017, the European activities on the development of nuclear data for fusion were integrated into the PPPT programme focusing on the needs of the PPPT neutronics supporting the development of the DEMO fusion power plant and the IFMIF-DONES neutron source. Transversal actions on Nuclear Data Evaluations (NDE) were implemented in the PPPT projects PMI (Plant Management and Integration), BB (Breeding Blanket), SAE (Safety and Environment), ENS (Early Neutron Source) and MAT (Materials).

The NDE tasks include general purpose neutron cross section data evaluations (ENDF) up to $200 \mathrm{MeV}$ incident neutron energy including co-variance data, special purpose nuclear data evaluations and libraries (neutron activation, displacement damage, gas production, deuteron cross-sections), specific method and tools developments, testing and benchmarking of the data by means of $\mathrm{V} \& \mathrm{~V}$ (verification and validation) and S/U (sensitivity and uncertainty) analyses. The tasks are conducted by Research Units (RU) of the European Union and Switzerland providing the expertise required for the different areas: CCFE (Culham, UK), IFIN-HH (Bucharest, Romania), JSI (Ljubljana, Slovenia), KIT (Karlsruhe, Germany), PSI (Würenlingen, Switzerland), TUW (Vienna, Austria), UNED (Madrid, Spain), and UU (University of Uppsala, Sweden).

The data evaluations provided with these activities are fed into the JEFF (Joint Evaluated Fission and Fusion) nuclear data libraries, maintained and disseminated by the NEA Data Bank of the OECD, Paris [3]. JEFF addresses the needs of the European nuclear fusion and fission communities and serves, among others, as reference data library for PPPT nuclear analyses.

\section{Nuclear Data Evaluations - tasks and achievements}

The 2017/18 PPPT work programme included activities on the evaluation of general purpose neutron cross-section data for the ${ }^{180,182,183,184,186} \mathrm{~W},{ }^{56} \mathrm{Fe}$ and ${ }^{16} \mathrm{O}$. The $\mathrm{W}$ evaluations aimed at providing full ENDF data files with covariance data up to $200 \mathrm{MeV}$ neutron energy for the later use in application calculations. The activities on ${ }^{56} \mathrm{Fe}$ and ${ }^{16} \mathrm{O}$ included the elaboration of advanced evaluation methodologies and nuclear models with the production of trial evaluations that can be used for testing purposes.

\section{$3.1180,182,183,184,186 \mathbf{W}$ neutron cross-section data evaluations for general purpose applications}

These evaluations were performed by KIT applying a Bayesian approach taking into account experimental and nuclear model uncertainties [4]. The evaluation procedure consists of the following steps: (i) the calculation of crosssections, angular distributions for elastic scattering, energy distributions of emitted particles, and the calculation of covariance matrices for cross-sections, (ii) the processing of the data into an ENDF data file, (iii) the selection and analysis of experimental data for the subsequent combination with the results of calculations, (iv) the evaluation using experimental data, results of model calculations, and covariance information, (v) recording of the final data in ENDF-6 format, and (vi) checking of the ENDF data file.

The nuclear model calculations were performed with a modified version of the TALYS code [5] which employs an improved description of the pre-equilibrium emission of particles and clusters [6]. It is based on a modified version of Blann's geometry depend hybrid (GDH) model [7] and results in a better reproduction of the particle emission spectra [8]. The model parameters were properly estimated using measured data for individual tungsten isotopes.

Processing of the TALYS output data and preparation of the preliminary data in ENDF-6 format was performed with the TEFAL-1.9 code [9]. Resonance parameters were added at this stage from the JEFF-3.3 data library.

Covariance matrices were produced on the basis of the Unified Monte Carlo (UMC) approach proposed by D. Smith [10]. The calculation includes the following steps: (i) choice of the best set of parameters for the selected nuclear models, (ii) assessment of uncertainties of model parameters, (iii) Monte Carlo sampling of input data sets, (iv) the execution of calculations for the input data files obtained in the previous step, and (v) the calculation of covariance matrices.

The evaluation of cross-sections using experimental data, results of model calculations, and covariance information was performed using the generalized least-squares (GLS) method [11] as implemented in KIT's BEKED code package [12].

Fig. 1 shows, as an example, the neutron emission cross-section for natural $\mathrm{W}$ at $14 \mathrm{MeV}$ incident neutron energy compared to available experimental data and evaluations from other data libraries. A comprehensive comparison is provided in Refs. [13] and [14]. The W evaluations are currently under intensive benchmarking and are thus subject to further modifications before the final data files are produced.

\subsection{Hybrid R-Matrix approach for the evaluation of $\mathrm{n}+{ }^{16} \mathrm{O}$ neutron cross-section data}

This work is conducted by TUW, Vienna, with the objective to develop a computational approach which can be utilized to evaluate neutron cross-section data for light mass nuclides such as ${ }^{9} \mathrm{Be},{ }^{12} \mathrm{C}$, or ${ }^{16} \mathrm{O}$, which are of high importance to fusion applications. The focus of the work within the 2017/18 PPPT work programme was on the development of an adapted R-matrix approach for the $n+{ }^{16} \mathrm{O}$ reaction system with the production of a prototype ENDF data file which can be used for test applications [15]. 


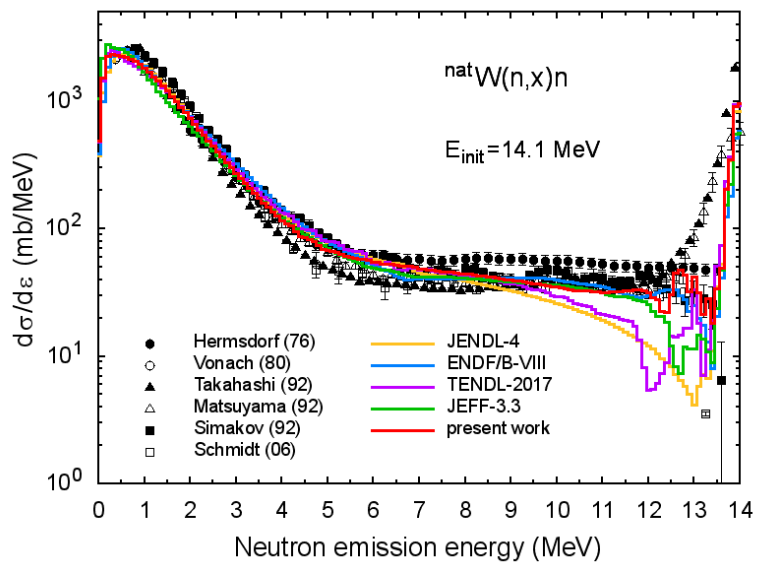

Figure 1. Neutron emission spectra for natural $\mathrm{W}$ at $14 \mathrm{MeV}$ incident neutron energy

The unitary hybrid R-matrix method, developed recently by TUW [16] was used to describe the available neutron induced reaction cross-sections of ${ }^{16} \mathrm{O}$ up to 13 $\mathrm{MeV}$ incident neutron energy. Beyond this incident neutron energy, the nuclear statistical model provides a fair description of almost all reaction channels. Hence a smooth transition between the two models is achieved enabling the generation of a complete evaluation of neutroninduced reaction data for ${ }^{16} \mathrm{O}$ up to $200 \mathrm{MeV}$.

The 2017/18 works involved the following steps: (i) development of a module in TUW's GECCCOS code system to optimise the statistical model calculations with TALYS; (ii) retrieval of experimental neutron-induced reaction data of ${ }^{16} \mathrm{O}$ at incident neutron energies higher than $6 \mathrm{MeV}$; (iii) statistical model calculations to find an optimized model which describes the available experimental data between $11 \mathrm{MeV}$ and $200 \mathrm{MeV}$; (iv) implementation of a more efficient fitting algorithm for the optimization of the R-matrix presentation; (v) optimization of the parameters of the R-matrix representation using the available experimental neutron-induced reaction data up to 13 $\mathrm{MeV}$; (vi) combining the results of (iii) and (v) to generate the prior of the envisaged Bayesian evaluation procedure; (vii) development of a concept for a unified Bayesian evaluation procedure over the whole energy range (i. e. resonance and statistical energy range) accounting for model defects and providing the co-variance matrices; (viii) generation of an ENDF file of the prior, starting point of the complete evaluation of neutron-induced reactions of ${ }^{16} \mathrm{O}$ up to incident neutron energies up to $200 \mathrm{MeV}$.

A prototype ENDF data file was produced for ${ }^{16} \mathrm{O}$ comprising the R-matrix generated data in the energy range up to $12 \mathrm{MeV}$ and statistical model based data, generated with TALYS calculations, up to $200 \mathrm{MeV}$ with a continuous transition at $12 \mathrm{MeV}$. Fig. 2 shows the resulting total cross-section. It is noted that besides the total cross sections also elastic differential cross sections are very well reproduced over the whole energy range.

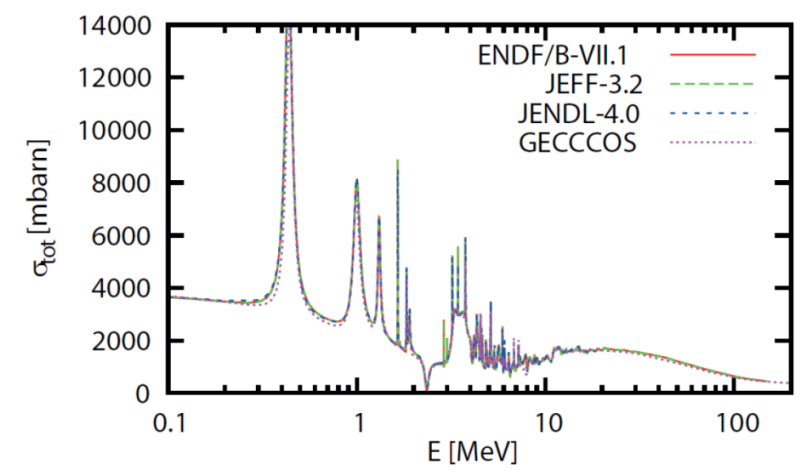

Figure 2. Evaluated total cross-section of $n+{ }^{16} \mathrm{O}$ (GECCCOS R-matrix calculation vs. evaluated data from major libraries

\subsection{Advanced methodologies for the evaluation of fast $\mathbf{n}+{ }^{56} \mathrm{Fe}$ neutron cross sections}

This activity, jointly conducted by PSI, Würenlingen, Switzerland, and the University of Uppsala, Sweden, aims at providing, on the example of ${ }^{56} \mathrm{Fe}$ neutron cross-section data, up-to-date methods for general purpose cross-section data evaluations for inclusion in nuclear data libraries. This includes an extension of the Total Monte Carlo (TMC) [17] approach with the Bayesian MC (BMC) method to find the best final evaluation, and the use of Gaussian processes to take into account model defects [18].

With the extended TMC approach not only model parameters are randomized but also nuclear models as implemented e. g. in the TALYS [5] and the EMPIRE [19] codes. In this work, 18,000 random files were produced for $\mathrm{Fe}-56$ which then were subjected to a Bayesian selection process using the BMC/BFMC (Backward-Forward MC) method [20]. Fig. 3 shows the example of the ${ }^{56} \mathrm{Fe}(\mathrm{n}, 2 \mathrm{n})$ cross-section with prior and posterior, and the considered experimental data.

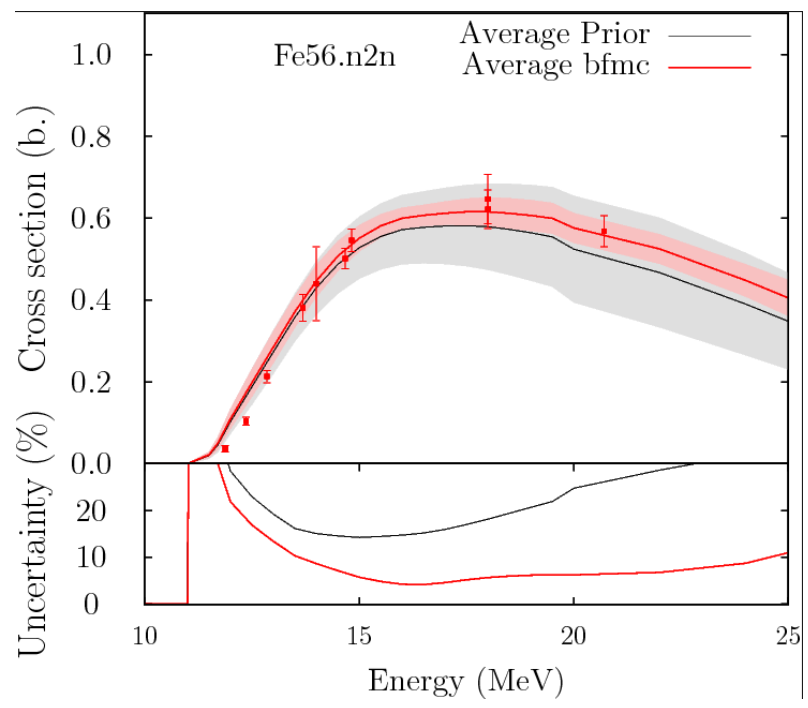

Figure 3. Prior and posterior cross-section for the ${ }^{56} \mathrm{Fe}(\mathrm{n}, 2 \mathrm{n})$ reaction evaluated on the basis of the BFMC method 
Such a procedure can be utilized for the evaluation of all neutron cross-section data resulting in an ENDF data file with co-variance data. However, this approach cannot overcome the existing limitations of the applied nuclear models. To account for such deficiencies, the concept of model defects based on Gaussian processes is considered as being developed by the University of Uppsala (UU).

In this approach, model defects are simulated through energy-dependent parameters in the TALYS code. The resulting parameter functions are modelled as Gaussian processes which are fitted together with the energyindependent parameters during the evaluation procedure [21]. The approach implemented for ${ }^{56} \mathrm{Fe}$ included the optimization of the TALYS parameters through an iterative scheme based on the Levenberg-Marquardt (LM) [22] fitting algorithm and the construction of a prior covariance matrix for the model parameters which can have an energy dependence.

The results obtained with this procedure for the ${ }^{56} \mathrm{Fe}$ reaction cross-sections in the fast neutron energy domain up to $30 \mathrm{MeV}$ were processed with the TEFAL code to produce the final ENDF data file including covariance matrices. Fig. 4 shows examples of produced ${ }^{56} \mathrm{Fe}$ neutron reaction cross-sections with uncertainties.
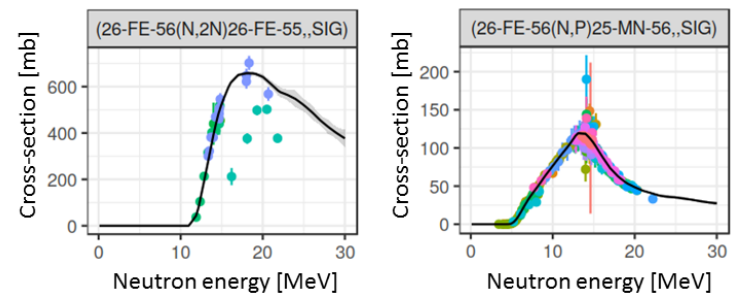

Figure 4. Evaluated and experimental ${ }^{56} \mathrm{Fe}$ neutron crosssections

Work is progressing to interface UU's model defect ansatz with PSI's extended TMC approach for the production of a full data evaluation with all uncertainty information and the final integration into a general purpose nuclear data library like JEFF. This is to be demonstrated for $\mathrm{n}+$ ${ }^{56} \mathrm{Fe}$ neutron cross-section data up to $200 \mathrm{MeV}$.

\subsection{Benchmark and validation analyses}

Benchmarking and validation are essential to check the quality of the evaluated nuclear data, identify potential deficiencies and shortcomings, and assess the performance for fusion applications, both in comparison to existing evaluations or model calculations, and experimental data. This is vital for the use of the data in design calculations or safety related analyses within the PPPT programme.

The current focus of the benchmarking activities is on the checking of the running data evaluations for oxygen, Fe, and W. Benchmark analyses are being conducted against FNS (Tokai-mura, Japan) experiments (liquid O slab, W slab), Fe spherical shells experiments (IPPE Obninsk, KfK - Karlsruhe, NPI Rez - $14 \mathrm{MeV}$ neutron source and Cf-252 fission neutron source), the Winfrith Iron Benchmark Experiment ("ASPIS IRON-88") with a fission spectrum source, the FNG (Frascati, Italy) W block experiment, and the Oktavian (University of Osaka) W spherical experiment.

Fig. 5 shows examples for reaction rates measured in the tungsten block experiment at the Frascati Neutron Generator (FNG) with $14 \mathrm{MeV}$ neutrons [23]. The curves labelled "KIT2019" show the results obtained with the current version of the $\mathrm{W}$ isotope evaluations presented in section 3.1.
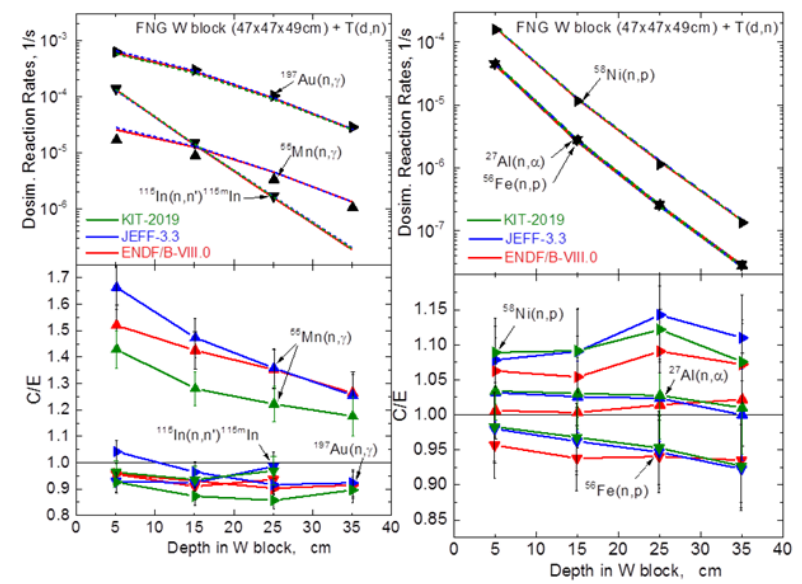

Figure 5. Profiles and $\mathrm{C} / \mathrm{E}$ (calculation/experiment) ratios for reaction rates measured in the FNG tungsten block experiment

\subsection{Activation cross-section data library}

Activation cross-section data are of highest importance to fusion applications since they form the basis for the calculation of the radioactivity inventories generated in the components/materials of the facility upon irradiation. The quality of activation cross-section data directly determines the accuracy of the predicted radiation sources and thus affects safety and licensing issues, decommissioning and waste management.

A major evaluation effort is therefore conducted in the European fusion programme on the production of a qualified activation data library for fusion inventory calculations. This has led to various versions of the European Activation File (EAF) with the latest version EAF-2010 [24] which terminated the EAF series of activation data libraries for fusion applications.

The strategy in the PPPT programme is to adopt the TENDL data library [25] as source data library for activation cross-sections. Significant efforts were thus undertaken to ensure that TENDL can actually preserve or increase the quality of EAF-2010 by including the variety of validated cross-sections and improving deficient data.

To this end, a dedicated effort is conducted in the PPPT programme to improve in TENDL reaction cross-sections of high importance for fusion activity inventory calculations. A set of 91 cross-sections, identified previously as 
deficient in the TENDL-2015 library [26] was thereby improved for the latest issue TENDL- 2017 [27]. Fig. 6 shows the example of the ${ }^{65} \mathrm{Cu}(\mathrm{n}, \gamma){ }^{66} \mathrm{Cu}$ activation crosssection which includes improved data in the resonance and the high energy range.

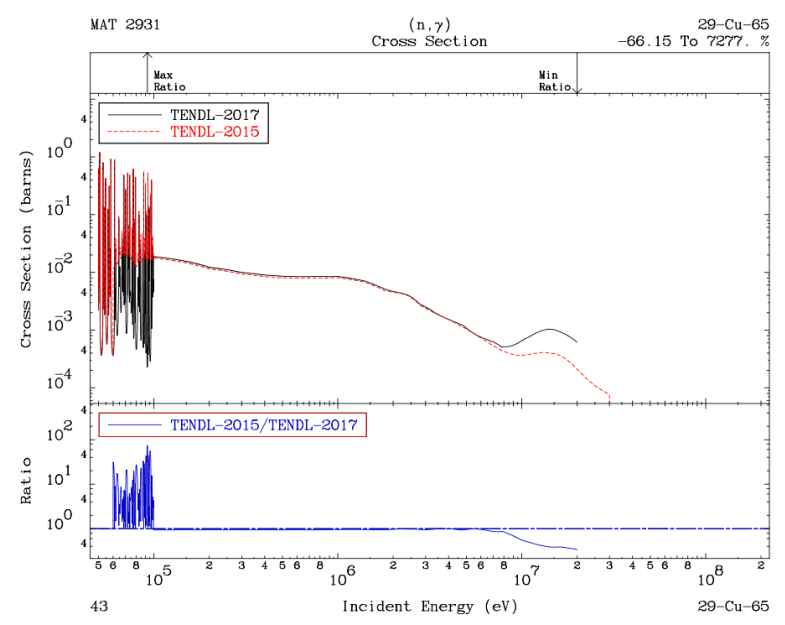

Figure 6. Improved ${ }^{65} \mathrm{Cu}(\mathrm{n}, \gamma){ }^{66} \mathrm{Cu}$ cross-section in TENDL2017

A dedicated activation data file was produced from TENDL-2017 by extracting and processing the activation cross-sections ("excitation functions") into the traditional EAF data format. This library was adopted as JEFF-3.3 activation data library [28] and, with some changes applied for light mass nuclides and actinides, was then processed into a 211 group data library which can be used by any activation code compatible with the EAF data format.

Comprehensive verification and validation $(\mathrm{V} \& \mathrm{~V})$ analyses were performed on both the activation data library in (groupwise) EAF format and the underlying TENDL-2017 data library. CCFE's validation data base was applied to this end including a wide range of differential and integral measurements, resonance integrals, thermal cross-sections, astrophysical data, and decay heat data [29], [30]. The $V \& V$ analyses were further complemented with statistical checks. Fig. 7 shows the C/E distribution of the integral checks performed on TENDL-2017 (ENDF format, 709 energy groups).

It is concluded that TENDL-2017 provides superior agreement with experimental data and is thus recommended as basis for the activation data library in fusion applications. In repeating the benchmark exercise on the derived TENDL-2017 activation data library in EAF format (211 groups) an overall good agreement was obtained. A few inconsistencies were though detected which are being currently fixed. With another V\&V analyses cycle successfully completed, the TENDL-2017 activation data library in EAF data format and 211 energy groups will be then ready to serve as (mandatory) reference library in the PPPT programme.

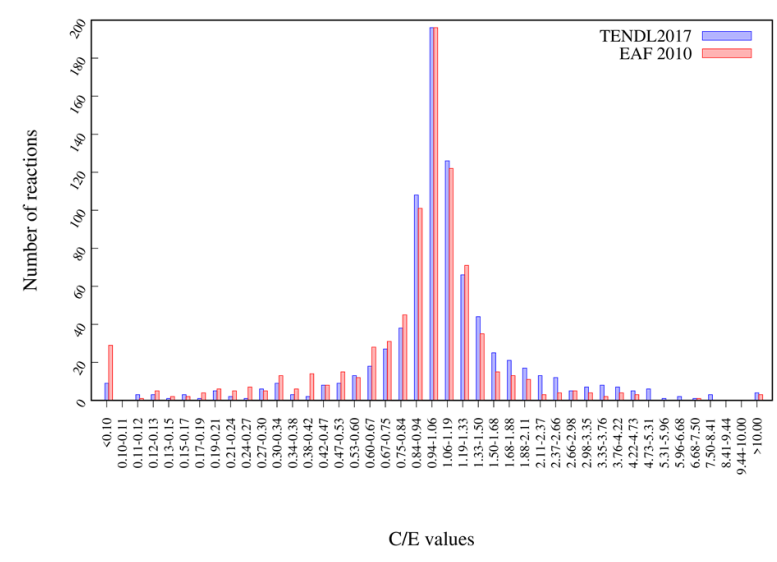

Figure 7. C/E ratios for all integral experiments used in the V\&V analyses of the TENDL-2017 data library

\subsection{Displacement damage cross-section data}

The assessment of neutron induced radiation damages builds on displacement cross-section data, in general based on the simple NRT damage model for the calculation of the number of lattice defects [31]. Specific dpa crosssections were prepared previously by A. Konobeev, KIT, for the calculation of the displacement damage of Eurofer and SS-316 steels. These cross-sections, available from the IAEA/NDS [32], are used as reference for the calculation of displacement damages to steel components in the PPPT programme.

The efforts to improve the data base and modelling capabilities for radiation damage calculations is continued within the PPPT programme with the extension of the NRT model to the athermal recombination corrected (arc) dpa formalism [33]. Arc-dpa cross-sections take into account lattice defects surviving thermal annealing and thus enable the estimation of the actual damage production in irradiated materials. The arc-dpa concept is a modification of the NRT formalism with additional parameters to describe the defect generation efficiency. These parameters can be derived from available experimental data, molecular dynamics simulation results and systematic inter- and extrapolations. This enabled the generation of a large set of arc-dpa cross-section data for the elements from $\mathrm{Li}$ to $\mathrm{U}$ utilizing neutron cross-section data from JEFF-3.3 and other major libraries [34]. Fig. 8 shows the example of the dpa cross-sections evaluated for tungsten.

The data are available in a sub-library of JEFF-3.3 [35] which includes dpa cross-sections prepared both with the NRT and the arc-dpa damage model. These cross-sections are recommended in the PPPT programme for the calculation of displacement damages of individual elements.

\subsection{Deuteron induced cross-section data}

High quality cross-section data of deuteron induced reactions are required for design analyses of the IFMIFDONES facility, developed as a neutron source for material irradiations within the ENS project of PPPT. The analyses include deuteron transport simulations, performed 


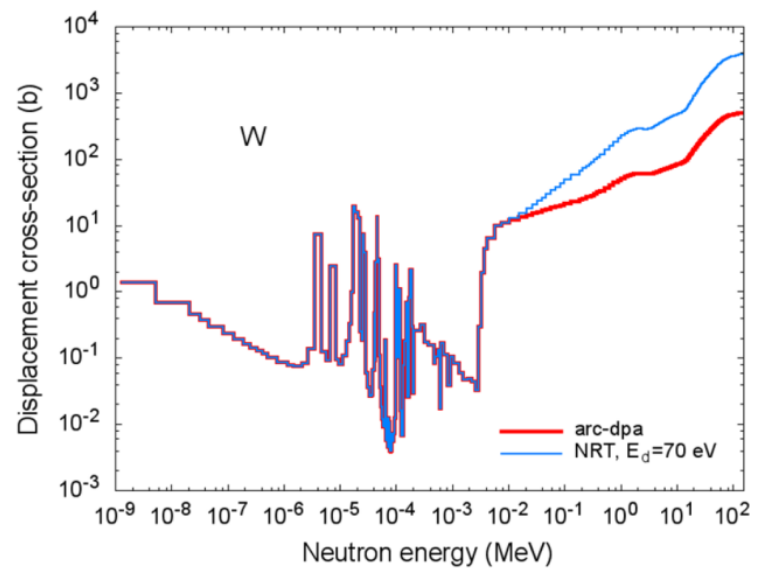

Figure 8. JEFF-3.3 displacement damage cross-section of tungsten calculated with arc-dpa and NRT damage models

primarily with the MCUNED Monte Carlo code [36] and activation calculations for accelerator components subjected to deuteron beam losses and secondary neutron radiation resulting from deuteron-induced reactions. The TENDL deuteron data library is the current reference used in the ENS project. The quality of the TENDL deuteron cross-sections, however, is in general poor since they are largely based on automated calculations with the TALYS code which employs reaction models that are not wellsuited for these reactions. This applies in particular to deuteron reactions on low mass nuclei.

A dedicated effort has been therefore allocated in the PPPT programme to improve the deuteron crosssection data for transport simulations and activation calculations. An advanced modelling approach was developed by M. Avrigeanu, IFIN-HH Bucharest, Romania, which takes proper account of the contributions from all involved reaction mechanisms including breakup, stripping, pick-up, pre-equilibrium and evaporation processes [37]. This was demonstrated with the recent evaluations of deuteron induced cross-sections for ${ }^{27} \mathrm{Al}$, ${ }^{50,52,53,54} \mathrm{Cr},{ }^{55} \mathrm{Mn},{ }^{54,56,57,58} \mathrm{Fe},{ }^{58,60,61,62,64} \mathrm{Ni},{ }^{63,65} \mathrm{Cu},{ }^{93} \mathrm{Nb}$ up to 60 deuteron energy $\mathrm{MeV}$ [38]. Fig. 9 provides an example for ${ }^{n a t} \mathrm{Cr}(\mathrm{d}, \mathrm{xn})$ and $(\mathrm{d}, \mathrm{pxn})$ cross-sections.

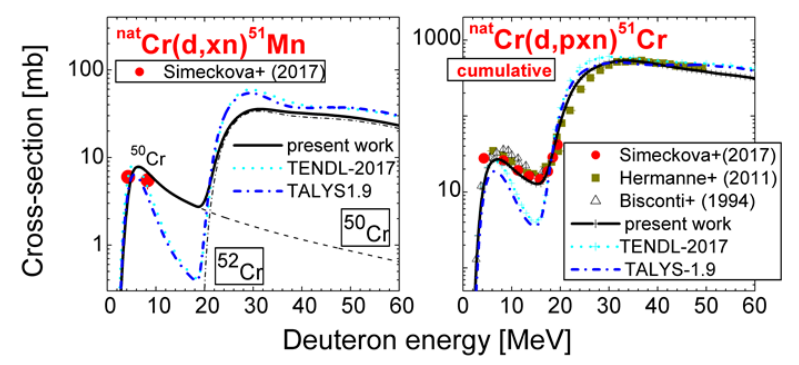

Figure 9. Measured and evaluated ${ }^{n a t} \mathrm{Cr}(\mathrm{d}, \mathrm{xn})$ and (d,pxn) crosssections taking into account break-up, stripping, pick-up, preequilibrium and evaporation processes[38]
This deuteron reaction model was implemented in an ad-hoc modified TALYS version 1.81. The strategy in the PPPT programme is to implement such a model in a future TALYS release and include improved deuteron crosssections in the next version of the TENDL data library.

\section{Summary andd conclusions}

EUROfusion's activities on the development of high quality nuclear data, implemented in the PPPT programme, were presented in this paper. The activities include nuclear data evaluations for neutron and deuteron induced reactions, the production of related data libraries, suitable for transport simulations, activation and radiation damage calculations which satisfy the needs for nuclear analyses of the DEMO fusion power plant and the IFMIF-DONES neutron source. The activities are closely linked to the JEFF initative of the NEA Data Bank, to which the produced data evaluations and libraries are provided.

The evaluation work is complemented by extensive benchmark, sensitivity and uncertainty analyses to check the performance of the evaluated cross-section data and libraries against integral experiments. This work will be further enhanced by inclusion in the PPPT programme dedicated experimental activities tailored to the needs of DEMO and IFMIF-DONES.

\section{Acknowledgment}

This work has been carried out within the framework of the EUROfusion Consortium and has received funding from the Euratom research and training programme 2014-2018 and 2019-2020 under grant agreement No 633053. The views and opinions expressed herein do not necessarily reflect those of the European Commission. The support by the NEA Data Bank, Paris, in providing their services for the data file assembly and maintenance, and hosting the progress meetings, is gratefully acknowledged.

\section{References}

[1] T. Donne, European roadmap to fusion energy, 30th Symposium on Fusion Technology, September 17-21, 2018, Giardini Naxos, Italy

[2] U. Fischer et al, Nuclear Data for Fusion Technology - The European Approach, EPJ Web of Conferences 146, 09003 (2017), https://doi.org/10.1051/epjconf/201714609003

[3] A. Plompen, The Joint Evaluated Fission and Fusion (JEFF) Nuclear Data Library, this conference

[4] A. Konobeyev , U. Fischer, P. Pereslavtsev, S. Simakov, New evaluation of general purpose neutron data for stable $\mathrm{W}$-isotopes up to $200 \mathrm{MeV}$, this conference

[5] A. Koning, D. Rochman, Modern nuclear data evaluation with the TALYS code system, Nucl. Data Sheets 113, 2841 (2012)

[6] A.Yu. Konobeyev, et al., Implementation of the geometry dependent hybrid model in TALYS, J. Korean Physical Society 59, 935 (2011) 
[7] M. Blann, Importance of the nuclear density distribution on pre-equilibrium decay, Phys. Rev. Lett. 28, 757 (1972)

[8] A. Konobeyev, U. Fischer, P. Pereslavtsev, A. Koning, M. Blann, Implementation of GDH model in TALYS1.7 code, KIT Scientific Working Papers 45, 2016, https://doi.org/10.5445/IR/1000052543

[9] A. Koning, TEFAL-1.9: Making nuclear data libraries using TALYS, Nuclear Research and Consultancy Group (NRG), November 2017

[10] D. L. Smith, A Least-squares computational tool kit, ANL/NDM-128, Argonne National Laboratory, 1993

[11] D. L. Smith, A Unified Monte Carlo Approach for to Fast Neutron Cross Section Data Evaluation, ANL/NDM-166, Argonne National Laboratory, 2008

[12] A. Konobeev, U. Fischer, P. Pereslavtsev, Computational Approach for the Evaluation of Nuclear Data Including Covariance Information, J. Korean Physical Society 59, 923 (2011)

[13] A. Konobeyev, U. Fischer, P. Pereslavtsev, S. Simakov, Evaluated data files for neutron irradiation of W-182 and W-186 at energies up to 200 $\mathrm{MeV}$, KIT Scientific Working Papers 108, 2019, https://doi.org/10.5445/IR/1000090132

[14] A. Konobeyev, U. Fischer, P. Pereslavtsev, S. Simakov, Evaluated data files for neutron irradiation of W-180 and W-183 at energies up to 200 $\mathrm{MeV}$, KIT Scientific Working Papers 123, 2019, https://doi.org/10.5445/IR/1000096730

[15] H. Leeb, Unified Bayesian Evaluation of Oxygen Based on the Hybrid R-matrix Method, this conference

[16] Th. Srdinko, H. Leeb, R-matrix approach at the intersection with the statistical model regime, EPJ Web of Conferences146, 12030 (2017), https://doi.org/10.1051/epjconf/201714612030

[17] A.J. Koning and D. Rochman, Towards sustainable nuclear energy: Putting nuclear physics to work", Ann. Nucl. Energy 35, 2024 (2008)

[18] G. Schnabel, Interfacing TALYS with A Bayesian Treatment of Inconsistent Data and Model Defects, this conference

[19] M. Herman, R. Capote, et al., EMPIRE: Nuclear Reaction Model Code System for Data Evaluation, Nucl. Data Sheets 108, 2655-2715 (2007)

[20] D. Rochman, E. Bauge, A. Vasiliev, H. Ferroukhi, et al., Monte Carlo nuclear data adjustment via integral information, Eur. Phys. J. Plus 133, 537 (2018), https://doi.org/10.1140/epjp/i2018-12361-X

[21] P. Helgesson, H. Sjöstrand, Treating model defects by fitting smoothly varying model parameters, Ann. of Nucl. Energy 120, 35-47 (2018) https://doi.org/10.1016/j.anucene.2018.05.026

[22] D. W. Marquardt, An Algorithm for Least-Squares Estimation of Nonlinear Parameters, Journal of the
Society for Industrial and Applied Mathematics 11, 431-441 (1963), https://doi.org/10.1137/0111030

[23] P. Batistoni, M. Angelone, L. Petrizzi, M. Pillon, Neutronics benchmark experiment on tungsten, J. Nucl. Mater. 329-333, 683-686 (2004)

[24] J.-Ch. Sublet, L. W. Packer, J. Kopecky, R. A. Forrest, A. J. Koning, D. A. Rochman, The European Activation File: EAF-2010 neutron-induced cross section library, CCFE-R(10)05, Culham Centre, UK, 2010

[25] D. Rochman, A.J. Koning, J. Ch. Sublet, et al., The TENDL library: Hope, reality and future, EPJ Web of Conferences 146, 02006 (2017), https://doi.org/10.1051/epjconf/201714602006

[26] N. Dzysiuk, A. Koning, D. Rochman and U. Fischer, Improving activation cross sections for fusion applications, Fus. Sci. and Technology 73,13-24 (2018) httpps://doi.org/10.1080/15361055.2017.1372682

[27] TENDL-2017 library, https://tendl.web.psi.ch/tendl_2017/tendl2017.html

[28] JEFF-3.3 activation sub-library, http://www.oecdnea.org/dbdata/jeff/jeff33/\#neutron

[29] M. Fleming, J.-Ch. Sublet, M. R. Gilbert, A. Koning and D. Rochman, TALYS/TENDL verification and validation processes: Outcomes and recommendations,EPJ Web of Conferences 146, 02033 (2017), https://doi.org/10.1051/epjconf/201714602033

[30] M. R. Gilbert and J.-Ch. Sublet, Experimental decayheat simulation-benchmark for $14 \mathrm{MeV}$ neutrons \& complex inventory analysis, Nucl. Fusion 59,086045 (2019) https://doi.org/10.1088/1741-4326/ab278a

[31] M. J. Norgett, M. T. Robinson, I. M. Torrens, A proposed method of calculating displacement dose rates, Nucl. Eng. Des. 33,50 (1975)

[32] Eurofer and SS-316 displacement cross-section, https://www-nds.iaea.org/public/download-endf/DXS/

[33] K. Nordlund et al, Primary Radiation Damage in Materials, Report NEA/NSC/DOC(2015)9, OECD 2015

[34] A Konobeyev, U. Fischer, S.P. Simakov, Atomic displacement cross sections for neutron irradiation of materials from $\mathrm{Be}$ to $\mathrm{Bi}$ calculated using the arc-dpa model, Nucl. Eng. and Technology 51, 170-175 (2018), https://doi.org/10.1016/j.net.2018.09.001

[35] JEFF-3.3 dpa sub-library, http://www.oecdnea.org/dbdata/jeff/jeff33/\#dpa

[36] P. Sauvan, J. Sanz, F. Ogando, MCUNED - MCNPX Extension for Using light Ion Evaluated Nuclear Data library, NEA Data Bank, Paris, NEA-1859/1 (2013)

[37] M. Avrigeanu, V. Avrigeanu, Role of breakup and direct processes in deuteron-induced reactions at low energies, Phys. Rev. C92, 021601 (2015)

[38] M. Avrigeanu, Consistent Assessment of Deuteron Interactions at Low and Medium Energies, this conference 